
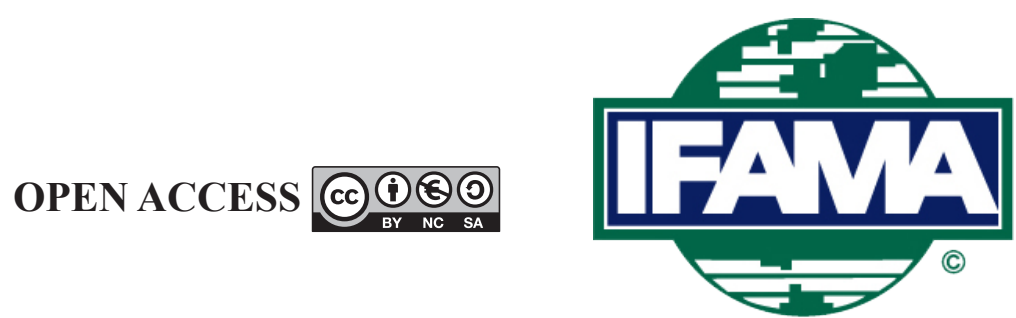

International Food and Agribusiness Management Review

Volume 23, Issue 5, 2020; DOI: 10.22434/IFAMR2019.0122

Received: 30 July 2019 / Accepted: 2 December 2019

Special issue: New and sustainable food and agribusiness management models

\title{
Market simulation of traceable food in China based on conjoint-value analysis: a traceable case of pork
}

RESEARCH ARTICLE

\author{
Bo Hou ${ }^{\mathrm{a}}$, Linhai $\mathrm{Wu}^{\oplus \mathrm{b}}$ and Xiujuan $\mathrm{Chen}^{\mathrm{c}}$ \\ ${ }^{a}$ Lecturer, School of Philosophy and Public Administration, Jiangsu Normal University, No. \\ 101, Shanghai Road, Tongshan District, Xuzhou, Jiangsu Province 221116, China P.R. \\ ${ }^{b}$ Professor, Institute for Food Safety Risk Management \& Research and School of Business, \\ Jiangnan University, No. 1800, Lihu Road, Binhu District, Wuxi, Jiangsu 214122, China P.R. \\ ${ }^{c}$ Lecturer, School of Business, Jiangnan University, No. 1800, Lihu \\ Road, Binhu District, Wuxi, Jiangsu 214122, China P.R.
}

\begin{abstract}
The food-traceability system is considered to be one of the main measures to fundamentally prevent foodsafety problems. It is of great value to study consumer demand for traceable food and market simulations that can adjust the production and supply structure of traceable food and promote traceable market development. Having sampled and interviewed 2,121 consumers in China, consumer preferences for traceable pork with different levels of safety information, and the respective market share of traceable pork with different product profiles were investigated using conjoint value analysis and the randomized first choice method. Results showed that Chinese consumers prioritized the certification of a traceable-pork safety-information attribute. Furthermore, consumers were willing to pay extra costs in order to obtain traceable safety information. However, this additional expenditure should account for no more than $30 \%$ of the price of ordinary pork, or consumer demand for safe pork decreases. Results of a market simulation also identified a type of traceable pork that had an optimal combination of attributes and met the needs of Chinese consumers. Consequently, the government should gradually promote a multilevel traceable-food market system in China by developing a combination of a certification mechanism and traceability system, and increasing financial subsidies for the construction of the traceability system.
\end{abstract}

Keywords: traceable safety information, consumer preferences, conjoint value analysis, market simulation JEL code: Q18

(1)Corresponding author: wlh6799@126.com 


\section{Introduction}

China is the world's largest producer and consumer of pork; moreover, China has been the world's largest pork producer for 30 consecutive years, and pork is the main type of meat consumed in China. In 2018, China's pork production reached 55.4 million tons, accounting for $49.25 \%$ of the total global pork consumption (Yin et al., 2018). However, as the most popular meat protein in China, pork also frequently suffers from safety incidents. A study by Hou (2018) showed that 14,583 pork-safety incidents occurred in China in 2006-2015 (Figure 1), out of which the major safety concern regarding pork products was the fact that pigs that had died from diseases were being sold to consumers. This type of incident, which accounted for $11.07 \%$ of the total number of pork-safety incidents, is a significant danger to public health and highlights the relevance and importance of establishing a pork-traceability system.

The Sanlu milk powder case, which occurred in September 2008, was a major food-safety incident in China and, once it was uncovered, the Ministry of Commerce and the Ministry of Finance of the People's Republic of China selected 54 cities nationwide to serve as pilot cities with an established meat-traceability system. China also implemented its New Food Safety Law in October 2015, which further clarified the required processes for the establishment of a food traceability system and provided legal protection for such actions. However, poor supply chain practices that have been happening for many years highlighted that China's traceable pork market is yet to achieve any substantive development (Wu et al., 2018).

Essentially, whether the development of a traceable pork market is sustainable depends on the needs of domestic consumers. However, since most Chinese consumers are not familiar with traceable food, which is in its infancy in China, it is difficult to obtain large amounts of actual purchase data and real demand data. In the field of food safety economics, scholars have studied consumer preferences for food with traceable attributes (Nguyen et al., 2018; Wu et al., 2017), food with animal welfare information (Olynk et al., 2010), animal food with bacterial infection risk information (Teisl and Roe, 2010), animal organic food (Van Loo et al., 2011), infant milk (Yin et al., 2018), and genetically modified foods (Carlsson et al., 2007).

The differences between the present study and the above studies are as follows. First, existing studies on traceable food do not subdivide the safety information by supply-chain processes, and traceable information is not certified. This is not conducive to examining the perceptions of different consumers regarding risks in different supply chain processes on certification preferences. Second, existing studies tended to calculate marginal willingness to pay for different attributes of the food profile. As consumers' overall willingness to pay for the food profile is not a simple sum of willingness to pay for each attribute, the study of marginal willingness to pay for attributes is of no practical significance to suppliers and to the development of related market policies. Third, the current international literature on attribute settings and food product levels does not reflect the domestic situation in China; thus, whether any research findings can be universally applied here and to similar countries needs to be further tested. For example, many foreign researchers believe that pig breeding methods are the most important attribute in food safety (Furnols et al., 2011). However, the recent occurrence of pork safety incidents has once again showed that breeding is clearly the weakest

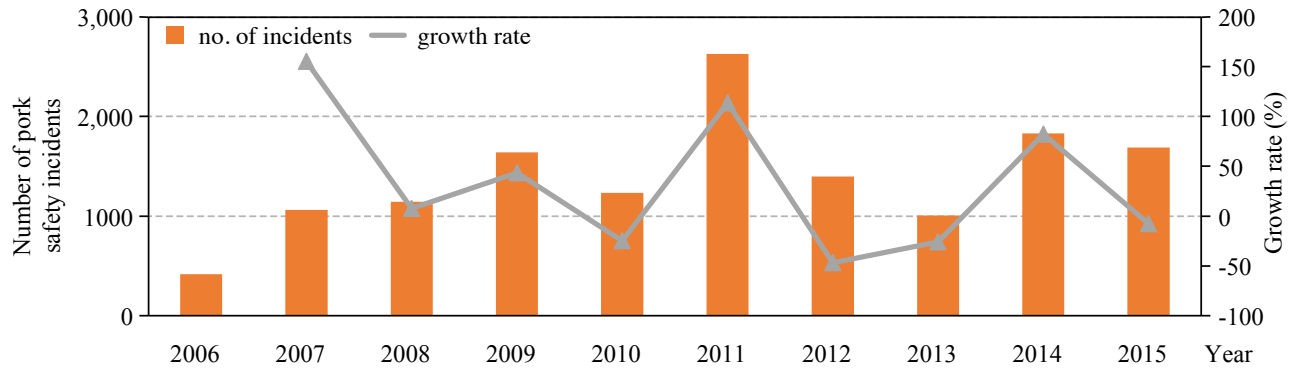

Figure 1. The total number of pork-safety incidents during 2006-2015. 
link in China's pork supply chain system and is associated with the highest level of food safety risk ${ }^{1}$. In this study, a traceable pork profile was considered as a whole, and the total market share of each traceable pork hindquarter profile was estimated through market simulation to explore the basic pathways for the development of pork traceability systems in China.

\section{Materials and methods}

\subsection{Method choice}

This paper presents a case study of traceable pork and discusses the basic pathways for the development of pork traceability systems in China by analyzing consumer preferences for traceable pork with different attribute and level combinations. Theoretically speaking, consumer preferences determine utility levels, which further affect consumers' payment behavior (Jehle and Reny, 2001), with the stated preference and revealed preference representing two basic methods for studying consumer preferences. Currently, international researchers mainly use the contingent valuation method (CVM), conjoint analysis (CA), and choice experiment (CE) to study stated preferences. However, a strategic bias that exaggerates consumers' willingness to pay (WTP) for products is likely to arise, partly due to the hypothetical nature of the CVM (Nguyen et al., 2018). Furthermore, it is difficult to simulate marketing plans for safe foods that are acceptable to consumers. Although the CE can be used to further decompose consumer preferences for safe foods in terms of their main attributes and levels, it requires consumers to make a choice from preset product profiles with the total price displayed. As a result, bias in experiment results are likely to occur due to consumers' insensitivity to the price of virtual profiles (Ding and Huber, 2009).

Based on the Generalized Axiom of Revealed Preference, empirical economics researchers tend to study consumer preferences by analyzing their payment behavior with commonly used methods, including the experimental auction and real choice experiment. Experimental auction and real choice experiment simulate a market trading environment in a realistic setting, and apply bidding mechanisms and the actual payment to promote incentive compatibility; therefore, both could overcome hypothetical experiment and social desirability biases (Ginon and Combris, 2014). However, the experimental auction is complicated and costly to use (Hobbs et al., 2005), whereas the real choice experiment might cause consumers to make nonoptimal tradeoff decisions between price and attribute preference (Ginon and Combris, 2014).

Since the 1970s, CA, a type of decomposing technology, has been used in the field of food product marketing research. This method provides consumers with a series of product profiles, and then the consumers give scores, provide a preference order, or make choices according to the characteristics of product profiles with different attribute and level combinations. Moreover, the use of ordinary least squares regression, Bayesian hierarchical models and a finite mixture could help quantify preference parameters associated with different attribute and level combinations (Mennecke et al., 2007). Furthermore, to date, CA has been developed into a powerful analytical tool that includes various quantitative methods, such as the full profile approach, adaptive conjoint analysis, conjoint value analysis (CVA), and choice based conjoint analysis. When the total number of concerned attributes does not exceed 6 and there is a relatively small sample size, CVA has a unique advantage (Huang, 1999). Since a response to a questionnaire can be presented as either a single concept or pairwise task, the latter can simultaneously provide two product profiles when the respondents are comparing products.

Such a comparison process could help respondents tell the differences between product attributes, and thus lead to more information being obtained. However, when compared with other research methods, studies that use CVA to analyze consumers' preference for food attributes and their WTP are very limited; therefore, the advantages of CVA in this research field has not been fully recognized. In particular, the extant literature does not consider the advantages of using CVA to estimate the market share of food items in the same category

\footnotetext{
${ }^{1}$ Statistics show that, during the period of 2006-2015, the number of pork-safety incidents in breeding, slaughtering and processing, sales was 5,556, 5,335 , and 3,692, respectively, accounting for $38.10 \%, 36.58 \%$, and $25.32 \%$ respectively (Hou, 2018; Yin et al., 2018).
} 
but with different attribute levels (Krystallis and Ness, 2005). By applying CVA, this paper provides a case study of traceable pork, tests consumer preferences for traceable pork with different attribute and level combinations in the context of the actual situation in China and discusses the basic pathways for the development of pork traceability systems in China.

\subsection{Attribute and level settings}

Given that consumer preferences for different parts of traceable pork might differ, the present study focuses on traceable pork hindquarters in order to eliminate the interference of nonessential factors. The traceability system of complete pork products should contain safety information related to the place of a product's origin, animal welfare, and product certification in quality and safety. Meanwhile, important information associated with major aspects of pork production, processing, and circulation should also be included (Wu et al., 2012a). As far as pork production is concerned, the entire supply chain of the pork production industry should include a wide variety of factors, such as boars, piglets, breeding, slaughtering, processing, transportation, and sales. The validity of any type of traceability depends on whether the settings of information attributes can completely cover all critical risk points of the supply chain system. A study by Wu et al. (2015) showed that the main safety risks of pork products in China are found across the entire supply chain system, and are related to breeding, slaughtering, transportation, and sales processes.

Therefore, based on the actual situation in China, three attributes of traceable pork, namely, price, traceable safety information, and the certification of traceable safety information, as well as the respective levels, were set in this paper (see attributes and level settings in Table 1). Specifically, the safety information attribute and level settings of traceable pork include three types of information, namely, breeding, slaughtering, and transportation. The certified traceable safety information attribute and level settings include the three levels proposed by Huang (1999), namely, noncertified, certified by an independent third party, and certified by a government organization. Using ordinary pork as reference, the price levels of traceable pork were estimated and set up by increasing these prices, which could not only help authors to better study consumers' WTP for traceable pork hindquarters, but also avoid research bias if unified prices were assigned to different cities.

Table 1. The settings of traceable pork with different attributes and levels.

\begin{tabular}{|c|c|c|}
\hline Attribute & Level and symbol & Description \\
\hline Price & $\begin{array}{l}\text { The price is increased by } 1-10 \%\left(\mathrm{X}_{1}\right) \\
\text { The price is increased by } 10-20 \%\left(\mathrm{X}_{2}\right) \\
\text { The price is increased by } 20-30 \%\left(\mathrm{X}_{3}\right) \\
\text { The price is increased by } 30-40 \%\left(\mathrm{X}_{4}\right)\end{array}$ & $\begin{array}{l}\text { The prices in percentage that respondents were } \\
\text { willing to pay for } 500 \mathrm{~g} \text { of the chosen pork. }\end{array}$ \\
\hline \multirow[t]{2}{*}{$\begin{array}{l}\text { The certification } \\
\text { of traceable safety } \\
\text { information }\end{array}$} & $\begin{array}{l}\text { Traceable information certified by a } \\
\text { government organization }\left(\mathrm{X}_{5}\right) \\
\text { Traceable information certified by an } \\
\text { independent third party }\left(\mathrm{X}_{6}\right)\end{array}$ & $\begin{array}{l}\text { The products carried a certification label indicating } \\
\text { pork quality testing by the government, or a } \\
\text { domestic or international third party certification } \\
\text { body. The testing involved inspection and } \\
\text { quarantine, sensory testing, physical and chemical } \\
\text { testing, and diseased pork detection. }\end{array}$ \\
\hline & $\begin{array}{l}\text { Non-certified traceable information } \\
\left(\mathrm{X}_{7}\right)\end{array}$ & $\begin{array}{l}\text { Harmful substances and veterinary drug residues } \\
\text { contained in the products did not exceed the } \\
\text { maximum limits specified in relevant standards. }\end{array}$ \\
\hline $\begin{array}{l}\text { Traceable safety } \\
\text { information }\end{array}$ & $\begin{array}{l}\text { Traceable code displaying breeding, } \\
\text { slaughtering and transporting } \\
\text { information }\left(\mathrm{X}_{8}\right)\end{array}$ & $\begin{array}{l}\text { Specific farming information covers pig farm, } \\
\text { farming environment, feed, and veterinary drug; } \\
\text { information of slaughter and processing covers } \\
\text { slaughter time, and location of slaughter and } \\
\text { processing; information of transporting covers } \\
\text { transportation, and carrier. }\end{array}$ \\
\hline
\end{tabular}




\subsection{Conjoint value analysis questionnaire design}

The different attributes and levels of food products together constitute food product profiles. However, if consumers have to identify more than 15-20 profiles, they become bored (Rossi et al., 1996). Therefore, it is better to enhance the selection efficiency of consumers by assigning a minimum number of tasks (Wu et al., 2015). In this study, the attributes of traceable pork safety information with different levels contained 36 (i.e. $3 \times 3 \times 4$ ) combined profiles, and thus consumers would have had to make a choice from 1,260 sets. Obviously, such a task is difficult, if not impossible, to accomplish. Therefore, this study used the randomized method in the SSI web 7.0 operating system (Sawtooth Software Inc., Sequim, WA, USA) and designed multiple version questionnaires to reduce the impact of psychological factors or related survey questions on respondents; thus, design efficiency was improved. Subsequently, in this study, the number of tasks for each questionnaire version was 8 , with 10 versions of the highest D-efficiency being randomly selected. Figure 2 shows an example of a task using CVA. Each task (left and right) consisted of pork hindquarter profiles with different attribute and level combinations, which were presented to the respondents for the purpose of experimental selection. The respondents had to give scores using a 9-point scale (1 meant a strong preference for product profiles on the left, 9 meant a strong preference for product profiles on the right, and 5 meant no difference). Having completed the experiment, the respondents also had to provide some basic information, such as age, gender, education, income, their main concern when buying pork hindquarters, their satisfaction level with the current food safety situation, their awareness of clenbuterol incidents, and their understanding of traceable information, so that the possible impact of these relevant factors on consumer preferences could be further examined.

\subsection{Data collection}

As mentioned above, the Ministry of Commerce and the Ministry of Finance of the People's Republic of China selected 54 cities nationwide to serve as pilot cities with an established meat traceability system. In this study, five of these pilot cities², namely: (1) Dalian City, Liaoning Province; (2) Shijiazhuang City, Hebei Province; (3) Wuxi City, Jiangsu Province; (4) Yinchuan City, Gansu Province; and (5) Kunming City, Yunnan Province were selected as experiment sites. These five cities are located in the Northeast,

\footnotetext{
${ }^{2}$ The reason for the selection of pilot cities was that residents in these cities have a certain level of awareness regarding traceable pork, and there was thus a knowledgeable basis from which to conduct a relevant survey. If a survey were conducted in a city that did not have a traceability system, the investigators would have to explain the concept in detail to local consumers, which would not only increase the survey time, but also reliance of the results on the communication skills of the investigators when they were explaining the relevant concepts to respondents, which may lead to uncertainty in the survey results.
}

Task 1: Which of the following options do you like?
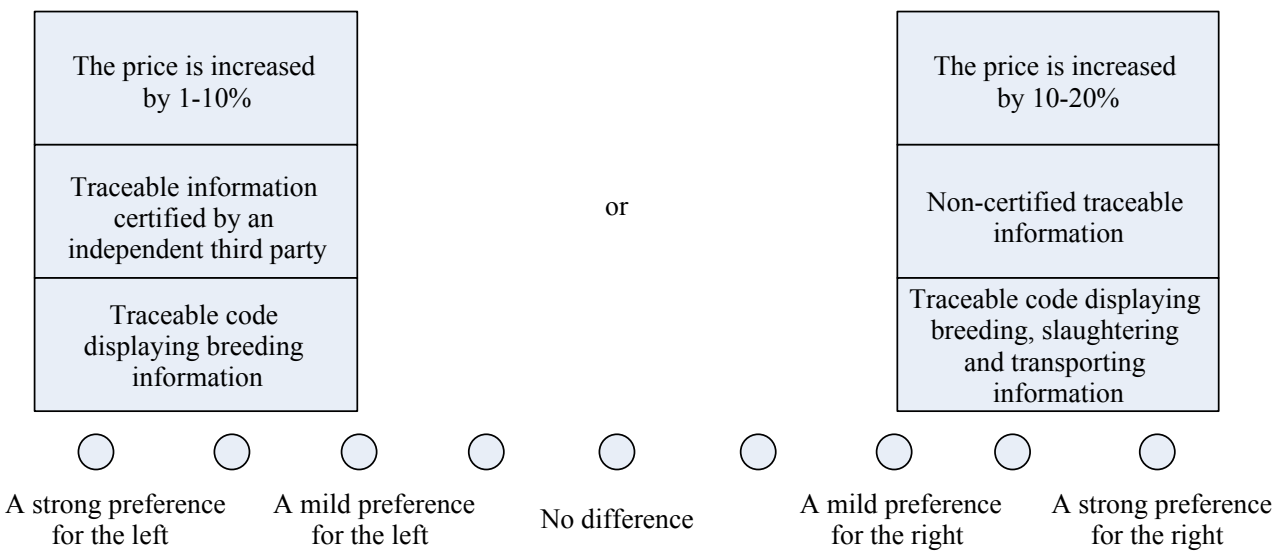

Figure 2. An example of a task using conjoint value analysis. 
North, East, Northwest, and Southwest of China, respectively. When surveys were conducted in these five cities, supermarkets, meat product stores, and farmer markets in urban areas, which are visited frequently, were specifically selected. This is because past experience has shown that these are the main channels for consumers looking to buy pork (Wu et al., 2012a). The survey was carried out by a trained investigator, who conducted one-on-one interviews with consumers (hereinafter referred to as respondents). In order to ensure the randomness of the survey samples, a method was chosen whereby the third customer who came into the investigator's field of view was selected as a respondent; this method was consistently adopted in the survey (Wu et al., 2012b). In this experiment, 550 questionnaires (10 different versions, with 55 copies for each version) were distributed in each of those five aforementioned pilot cities, with 519, 521, 527, 514 , and 528 returned copies, respectively. This gave a total of 2,609 questionnaires, of which 2,121 were valid with complete answers.

\section{Results}

\subsection{Brief descriptive analysis}

In total, $56.44 \%$ of respondents in this study were female, a proportion slightly higher than that of the males. The proportion of respondents in the 26-40 and $41-55$ age groups was $49.01 \%$ and $31.19 \%$, respectively, which represented the largest component of respondents. Those respondents who had a high school education or below were dominant, accounting for $64.36 \%$ of the overall sample. Respondents with a monthly income of RMB 2,000-5,999 accounted for $51.98 \%$ of the overall sample. $67.82 \%$ of the respondents had a child under the age of 18 , and $89.11 \%$ of the respondents were aware of clenbuterol incidents.

In addition, statistics showed that $57.43 \%$ of the respondents believed that the inclusion of traceable food information could help enhance food safety. Furthermore, $35.15 \%$ and $61.88 \%$ of the respondents had a fully confident or skeptical attitude, respectively, toward the authenticity of traceable information. The proportion of respondents who had a fully confident or skeptical attitude towards the authenticity of traceable information certified by the government was $66.34 \%$ and $29.21 \%$, respectively. By contrast, the proportion of respondents who had a fully confident or skeptical attitude towards the authenticity of traceable information certified by an independent third party was $35.15 \%$ and $53.47 \%$, respectively, suggesting a higher level of consumer trust in government certified traceable information. Of the respondents, $72.28 \%$ and $58.91 \%$ believed that information from breeding and slaughtering processes represented the most important and the second most important information relating to traceable pork safety, respectively, suggesting that consumer preferences for specific traceable information are relatively consistent.

\subsection{Results of conjoint value analysis model estimation}

Lancaster (1966) believed that consumer utility was derived from the attributes of a commodity rather than the commodity itself. In this study, traceable pork could be considered as a combination of several attributes, including traceable safety information, the certification of traceable safety information and price. Due to budget constraints, consumers have to choose an attribute combination of traceable pork in order to maximize their own utility. Through a thorough consideration of different attribute and level combinations of traceable pork, CVA can simulate different product profiles for consumers to select, which also meets the expected utility hypothesis (Ortega et al., 2011) developed by Lancaster (1966). Therefore, CVA is an appropriate method to simulate consumers' actual preference for traceable pork and their purchase decisions.

In Equation 1, $U_{i m t}$ represents the obtained utility and refers to the scenario that consumer $i$ selects traceable pork profile $m$ from the subset $J$ of choice space $C$ under situation $t . U_{i m t}$, consists of two parts (Ben-Akiva and Gershenfeld, 1998): the first part is the deterministic term $V_{i m t}$ and the second part is the stochastic term $\varepsilon_{i m t}$ :

$$
U_{i m t}=V_{i m t}+\varepsilon_{i m t}
$$




$$
V_{\text {imt }}=\beta_{i} X_{\text {imt }}
$$

in which $\beta_{i}$ is the part worth vector of consumer $i$, and $X_{i m t}$ is the attribute vector of traceable pork profile $m$.

If $\varepsilon_{i m t}$ follows a normal distribution without regard to the actual situation, then Equation 1 can be used as a full profile approach (FPA). However, since the FPA requires that all traceable pork profiles should be rankordered and scored, the total number of profiles is constrained. For example, Green and Srinivasan (1978) believed that the total number of profiles with FPA applied should be no more than 30 . Therefore, this study uses CVA based on grade pairwise comparisons, and respondents only had to make a choice between two traceable pork profiles. Therefore, the problem commonly found in the FPA, that the total number of profiles is constrained as a compromise when the effect of data collection is enhanced, can be solved satisfactorily. Let us assume that when the consumer selects traceable pork profile $m$ this is based on the consideration that $U_{i m t}>U_{\text {int }}$ always works as long as $n \neq m$. The utility difference between profile $m$ and $n$ could be expressed as the following linear regression equation:

$$
y_{i t}=U_{i m t}-U_{i n t}=\beta_{i} x_{i t}+\mu_{i t}
$$

in which $\Delta \mathrm{U}_{\mathrm{it}} \geq 0, \mu_{i t}=\varepsilon_{i m t}-\varepsilon_{i n t} \times x_{i t}=X_{i m t}-X_{\text {int }}$

Table 2 displays the regression results of the CVA model with the applied multiple least squares method. It shows that, as far the price attribute of traceable pork was concerned, it was statistically significant at the $1 \%$ level when prices were increased by $30-40 \%$, but statistically nonsignificant when prices were increased by $10-20 \%$ or $20-30 \%$. Therefore, consumers became aware of an increase in pork prices when they were increased by $30-40 \%$. For the certification of traceable pork safety information, only the level of noncertified traceable information was statistically significant at the $1 \%$ level, suggesting that consumer preferences for government certified products were significantly higher than noncertified products. The reason that the level of products certified by an independent third party was statistically nonsignificant was probably because consumers believed that there was no difference between products certified by a government organization and an independent third party. With regard to safety information attributes related to traceable pork, they should contain both breeding and slaughtering information, or just breeding information. Furthermore, both scenarios were statistically significant at the $5 \%$ level, indicating that consumer preferences for these two scenarios were significantly lower than for the scenario when all the information related to breeding, slaughtering, and transportation (i.e. the highest level) was included. Furthermore, if the range of scores for different attributes and levels of traceable pork were divided by the sum of the range of all attribute scores to serve as an importance index of each attribute, the relative importance of each attribute could be rank

Table 2. The utility values in different attribute and level combinations. ${ }^{1,2}$

\begin{tabular}{lllc}
\hline Level symbol & Part worth & Standard deviation & $P$-value \\
\hline $\mathrm{X}_{1}$ & 0.6066 & - & - \\
$\mathrm{X}_{2}$ & -0.1168 & 0.5822 & 0.3909 \\
$\mathrm{X}_{3}$ & -0.1758 & 0.5621 & 0.3798 \\
$\mathrm{X}_{4}$ & $-0.3140^{* *}$ & 0.0923 & 0.0012 \\
$\mathrm{X}_{5}$ & 1.6553 & - & - \\
$\mathrm{X}_{6}$ & -0.5720 & 0.7782 & 0.3044 \\
$\mathrm{X}_{7}$ & $-1.0833^{* *}$ & 0.0525 & $<0.0001$ \\
$\mathrm{X}_{8}$ & 0.3419 & - & - \\
$\mathrm{X}_{9}$ & $-0.1340^{*}$ & 0.0539 & 0.0182 \\
$\mathrm{X}_{10}$ & $-0.2079^{*}$ & 0.0834 & 0.0179 \\
\hline
\end{tabular}

$1 *$ statistically significant at the $5 \%$ level, **statistically significant at the $1 \%$ level.

${ }^{2} \mathrm{~F}=62.176>\mathrm{F}_{0.05}(62.114)=2.1028 ; \mathrm{R}^{2}=0.85 ;$ Adjusted $\mathrm{R}^{2}=0.72$. 
ordered as (from highest to lowest) certification of traceable safety information, price, and traceable safety information, with their relative importance as $65.07,21.87$, and $13.06 \%$, respectively.

\subsection{Traceable pork market simulation results}

Based on the above discussion, for each attribute of traceable pork, the first level has the highest part worth. Undoubtedly, consumers would most prefer traceable pork whose farming, slaughtering, and transporting information was certified by the government if the price was only increased by $1-10 \%$. Due to cost considerations however, manufacturers of traceable pork are not necessarily willing to supply traceable pork to the market with the aforementioned attribute and level combination. Therefore, the key to developing a traceable pork market is to provide traceable pork with certain attribute and level combinations that are favored by consumers and likely to be offered by manufacturers. In view of this, and given that there is no significant difference between government certification and third party certification, this study chose government certification as a comparison reference, developed six possible traceable pork profiles, as shown in Table 3, and provided eight market supply schemes. In Table 4, Schemes 1-4 belong to a government certified category, and Schemes 5-8 belong to a noncertified category. A basis explanation is now provided. Under a scenario in which traceable pork safety information is certified by the government, Scheme 1 refers to a situation where Types A and B traceable pork are simultaneously sold in the market. Similarly, Scheme 2 refers to a situation where Types B and C traceable pork are simultaneously sold in the market. The other schemes follow similar patterns.

In order to avoid any bias caused by independence from irrelevant alternatives (IIA), this study used the Randomized First Choice Method to calculate the corresponding market share for each specific market scheme. Table 4 shows that, for Schemes 1 and 2, if Types A and B traceable pork, or Types B and C traceable pork, are simultaneously sold in the market, then the market share of Type B is the highest, which is more than $50 \%$ for both schemes. The reason for this is that, when the price of Type $\mathrm{C}$ traceable pork goes beyond $30 \%$, consumers reduce their safety requirements for pork products and choose Type B instead. By contrast, if the price of Type A and B traceable pork is increased by no more than $30 \%$, consumers choose Type B, which is safer than Type A. Therefore, it is easy to see how the market share of Type A traceable pork is over $50 \%$ in Scheme 4.

It should be noted that, if Types A, B, and C traceable pork are sold in the market simultaneously (i.e. Scheme 3 in Table 4), the ranking order regarding the market share of traceable pork is (from highest to lowest) A, $\mathrm{B}$, and $\mathrm{C}$, with the corresponding values as $33.88,33.44$, and $32.68 \%$, respectively. Let us assume that the current market implements Scheme 1 first (i.e. Types A and B traceable pork are simultaneously sold in the

Table 3. The definition of traceable pork product profiles. ${ }^{1}$

\begin{tabular}{|c|c|c|c|c|c|c|}
\hline \multirow[t]{2}{*}{ Attribute levels } & \multicolumn{6}{|c|}{ Type of traceable pork } \\
\hline & A & B & $\mathrm{C}$ & $\mathbf{D}$ & $\mathbf{E}$ & $\mathbf{F}$ \\
\hline Breeding information displayed & $\sqrt{ }$ & & & $\sqrt{ }$ & & \\
\hline Breeding and slaughtering information displayed & & $\sqrt{ }$ & & & $\sqrt{ }$ & \\
\hline Breeding, slaughtering and transporting information displayed & & & $\sqrt{ }$ & & & $\sqrt{ }$ \\
\hline Government certified & $\sqrt{ }$ & $\sqrt{ }$ & $\sqrt{ }$ & & & \\
\hline Non-certified & & & & $\sqrt{ }$ & $\sqrt{ }$ & $\sqrt{ }$ \\
\hline Prices increased by $1-10 \%$ & & & & $\sqrt{ }$ & & \\
\hline Prices increased by $10-20 \%$ & $\sqrt{ }$ & & & & $\sqrt{ }$ & \\
\hline Prices increased by $20-30 \%$ & & $\sqrt{ }$ & & & & $\sqrt{ }$ \\
\hline Prices increased by $30-40 \%$ & & & $\sqrt{ }$ & & & \\
\hline
\end{tabular}

$1 \sqrt{ }$ refers to the attribute and level combinations for a specific type of traceable pork. 
Table 4. The estimation of the market share of government certified and non-certified traceable pork with different attribute and level combinations (\%).

\begin{tabular}{lllllllll}
\hline & \multicolumn{3}{c}{ Scheme } & \multicolumn{2}{c}{ Type of traceable pork } & \multicolumn{2}{c}{ Total } \\
\cline { 2 - 8 } & & A & B & C & D & E & F \\
\hline Government certified & 1 & 49.41 & 50.59 & - & - & - & - & 100 \\
traceable pork & 2 & - & 52.26 & 47.74 & - & - & - & 100 \\
& 3 & 33.88 & 33.44 & 32.68 & - & - & - & 100 \\
Non-certified traceable & 5 & 51.50 & - & 48.50 & - & - & - & 100 \\
pork & 5 & - & - & - & 50.23 & 49.77 & - & 100 \\
& 6 & - & - & - & - & 47.42 & 52.58 & 100 \\
& 7 & - & - & - & 32.97 & 30.44 & 36.59 & 100 \\
& 8 & - & - & - & 48.11 & - & 51.89 & 100 \\
\hline
\end{tabular}

market), and then switches to Scheme 3 (i.e. Types A, B, and C traceable pork are simultaneously sold in the market). According to IIA, the impact of Type $\mathrm{C}$ on the market share of Types $\mathrm{A}$ and $\mathrm{B}$ should be the same, but the simulation results of Scheme 3 do not hold and thus invalidate IIA. Indeed, the substitution effect of Type $\mathrm{C}$ traceable pork on Type $\mathrm{B}$ is higher, whereas Type A traceable pork could gain more of the market share than Type B.

Table 4 shows that, for the noncertification scenario, the price of Types D, E, and F traceable pork is no more than $30 \%$. According to the assumptions outlined above, consumers should be more concerned about the safety of traceable pork under this scenario. Schemes 6 and 8 also display this pattern, namely, the higher the level of traceable safety information is, the larger the corresponding market share; however, Schemes 5 and 7 are different. For example, the market share of Type D traceable pork containing a single piece of information is higher than that of Type E. Further analysis shows that, in Table 4, the safety information of Type E, rather than that of Type F, contains slaughtering information. This is similar to Scheme 1, where Type B traceable pork was more favored by consumers than Type A.

Moreover, the safety information of Type B traceable pork contains government certified slaughtering information that is absent in Type A, suggesting that most consumers tended to believe that this type of information does not necessarily enhance pork safety. Instead, they would select Type D traceable pork with a lower price. This is probably related to the fact that there has been an implementation of pork slaughtering rules at fixed locations in China for a long time; therefore, consumer trust in slaughtering information decreases if government certified slaughtering information is lacking.

\section{Conclusions and implications}

In this study, we set different levels for three attributes of traceable pork, namely, traceable information, the certification of traceable information, and price, and applied CVA to study consumer preferences for traceable pork with different safety attributes. On this basis, we further simulated the market share of traceable pork under two scenarios (i.e. with or without government certification). The main conclusions are as follows:

Consumers are most concerned about the certification of traceable pork safety information, followed by price and traceable safety information. Furthermore, with regard to the certification of traceable pork safety information, government certification has the highest utility value. The possible reason is that, in an emerging market, fake certification of traceable food safety information is a widespread phenomenon due to economic interests and a lack of supervisory mechanisms. As a result, consumer trust in the certification of traceable foods is generally not very high. 
Generally speaking, consumers are willing to pay a certain amount of extra costs for traceable pork with provided safety information. However, the extra cost threshold associated with safety information that consumers are willing to pay is $30 \%$. When extra costs are less than $30 \%$, consumers prefer traceable pork with higher safety levels; however, when extra costs go beyond $30 \%$, consumers have a lower demand for pork safety. In other words, a consumer's preference for traceable pork actually depends on trade off decisions between the attributes of price and safety, and it is likely that they could even ignore the safety attribute if the price is low enough. Furthermore, consumer price sensitivity is probably related to their income and consumption frequency.

Whether traceable pork is certified by the government or not also has an impact on its market share. When traceable pork is certified by the government, breeding information, slaughtering information, and a price increase of $20-30 \%$ all lead to an optimal combination of attributes. By contrast, in the absence of certification, breeding, slaughtering, and transporting information, and a price increase of $20-30 \%$ lead to an optimal combination of attributes, for which a key factor determining consumers' judgments of authenticity is whether slaughtering information is certified by the government.

Based on the above conclusions, there are two basic pathways that China can choose in the near future in order to develop a traceable pork market.

First, a local pork traceability system that is certified by the government should be established. This is because, if traceable pork is certified by the government, it is bound to increase its cost. However, once extra costs exceed the threshold acceptable to consumers, they lower their demand for food safety. Therefore, the development of a traceability system with partial safety information certified by the government should be an option. In order to further enhance food safety, it is also possible to increase government subsidies but decrease the relative price of traceable pork hindquarters. Consequently, due to the impact of the substitution and income effects, consumers increase their levels of expenditure. Therefore, a sharing mechanism of additional charges by consumers, manufacturers, and the government could be established; thus, a traceable pork system with all safety information certified by the government should be gradually developed.

Second, a comprehensive pork traceability system with a combination of noncertified information and certified local information labels should be established. Since the cost of a traceability system based on noncertified information is relatively low, the aspect of food safety can be fully taken into account, and a noncertified pork traceability system with all related covered safety information could be established. As for some key information, such as related to slaughtering, the auxiliary establishment of a certification and labeling system could be beneficial.

This study does have some limitations. For example, the experiment was conducted in Wuxi, a relatively developed city in China; therefore, research conclusions need further verification. At the same time, there is little literature on the CVA method for Chinese consumers, so this study lacks comparative analysis of research conclusions. However, it should be noted that, although the above shortcomings exist in this study, exploration of the CVA method and the basic conclusions based on the survey have scientific value for the development of the traceable food market in developing countries.

\section{Acknowledgements}

We acknowledge the supports from the National Social Science Foundation of China under Grant [17CGL044].

\section{Conflicts of interest}

The authors declare no conflict of interest. 


\section{References}

Ben-Akiva, M. and S. Gershenfeld. 1998. Multi-featured products and services: analysing pricing and bundling strategies. Journal of Forecasting 17(3-4): 175-196.

Carlsson, F., P. Frykblom and C.J. Lagerkvist. 2007. Consumer benefits of labels and bans on GM foods - choice experiments with Swedish consumers. American Journal of Agricultural Economics 89: 152-161.

Ding, M. and J. Huber. 2009. When is hypothetical bias a problem in choice tasks and what can we do about it? In: Proceedings of the fourteenth Sawtooth Software Conference. March 25-27, 2009. Delray Beach, FL, USA, pp. 263-272.

Furnols, M.F., C. Realini, F. Montossi, C. Sañudo, M.M. Campo, M.A. Oliver, G.R. Nute and L. Guerrero. 2011. Consumer's purchasing intention for lamb meat affected by country of origin, feeding system and meat price: a conjoint study in Spain, France and United Kingdom. Food Quality and Preference 22(5): 443-451.

Ginon, E. and P. Combris. 2014. What do we learn from comparing hedonic scores and willingness-to-pay data? Food Quality and Preference 33: 54-63.

Green, P.E. and V. Srinivasan. 1978. Conjoint analysis in consumer research: issues and outlook. Journal of Consumer Research 5(2): 103-123.

Hobbs, J.E., D. Bailey, D.L. Dickinson and M. Haghiri. 2005. Traceability in the Canadian red meat sector: do consumers care? Canadian Journal of Agricultural Economics 53(1): 47-65.

Hou, B. 2018. Research on consumer preference and public policy of traceable food. Social Sciences Academic Press, Beijing, China.

Huang, Z.R. 1999. A conjoint analysis on consumers' preferences to vegetable safety and certification. Journal of Agricultural Economics 66: 21-74.

Jehle, G.A. and P.J. Reny. 2001. Advanced microeconomic theory. Ashford Colour Press Ltd, Gosport, UK.

Krystallis, A. and M. Ness. 2005. Consumer preferences for quality foods from a South European perspective: a conjoint analysis implementation on Greek olive oil. International Food and Agribusiness Management Review 8(2): 62-91.

Lancaster, K. 1966. A new approach to consumer theory. Journal of Political Economy 74(2): 132-157.

Mennecke, B.E., A.M. Townsend, D.J. Haye and S.M. Longergan. 2007. A study of the factors that influence consumer attitudes toward beef products using the conjoint market analysis tool. Journal of Animal Science 85(10): 2639-2659.

Nguyen, H.D.M., D. Matty, J.V.L. Ellen, G. Annalyn, R. Pieter, H.T. Tran and V. Wim. 2018. What is the value of sustainably-produced rice? Consumer evidence from experimental auctions in Vietnam. Food Policy 79: 283-296.

Ortega, D.L., H.H. Wang, L.P. Wu and N.J. Olynk. 2011. Modeling heterogeneity in consumer preferences for select food safety attributes in China. Food Policy 36(2): 318-324.

Olynk, N.J., G.T. Tonsor and C.A. Wolf. 2010. Consumer willingness to pay for livestock credence attribute claim verification. Journal of Agricultural and Resource Economics 35(2): 261-280.

Rossi, P.E., R.E. McCulloch and G.M. Allenby. 1996. The value of purchase history data in target marketing. Marketing Science 15(4): 321-340.

Schnettler, B., R. Vidal, R. Silva, L. Vallejos, N. Sepúlveda. 2009. Consumer willingness to pay for beef meat in a developing country: The effect of information regarding country of origin, price and animal handling prior to slaughter. Food Quality and Preference 20(2): 156-165.

Teisl, M.F. and B.E. Roe. 2010. Consumer willingness-to-pay to reduce the probability of retail foodborne pathogen contamination. Food Policy 35: 521-530.

Van Loo, E.J., V. Caputo, R.M. Nayga, J.F. Meullenet and S.C. Ricke. 2011. Consumers' willingness to pay for organic chicken breast: evidence from choice experiment. Food Quality and Preference 22: 603-613.

Wu, L.H., F. Bu and D. Zhu. 2012a. Analysis of consumer preferences for traceable pork with different quality safety information. Chinese Rural Economic 10: 13-23.

Wu, L.H., L.L. Xu and D. Zhu. 2012b. Factors affecting consumer willingness to pay for certified traceable food in Jiangsu Province of China. Canadian Journal of Agricultural Economics 60(3): 317-333. 
Wu, L.H., S.S. Qin, D. Zhu, Q.G. Li and W.Y. Hu. 2015. Analysis of consumer preference for traceable pork origin attributes and traceable information attributes. Chinese Rural Economic 6: 47-62.

Wu, L.H., X.R. Gong, S.S. Qin, X.J. Chen, D. Zhu., W.Y. Hu and Q.G. Li. 2017. Consumer preferences for pork attributes related to traceability, information certification, and origin labeling: based on China's Jiangsu Province. Agribusiness 33(3): 424-442.

Wu, L.H., X.R. Gong, X.J. Chen and D. Zhu. 2018. Attribute with pre-incident quality assurance and postincident traceability. Chinese Journal of Population Resources and Environment 28(8): 148-160.

Yin, S.H, L. Rui, L.H, Wu and X.J. Chen. 2018. The 2018 China food safety development report. Peking University Press, Beijing, China. 